\title{
Niraparib: A Review in First-Line Maintenance Therapy in Advanced Ovarian Cancer
}

\author{
Arnold Lee ${ }^{1}$ (D) \\ Accepted: 1 September 2021 / Published online: 11 October 2021 \\ ๑) Springer Nature 2021, corrected publication 2021
}

\begin{abstract}
Niraparib (Zejula ${ }^{\mathrm{TM}}$ ) is a PARP inhibitor which is approved for maintenance therapy in adults with advanced ovarian cancer in complete or partial response to platinum-based chemotherapy. In a placebo-controlled phase III trial in patients with newly diagnosed advanced ovarian cancer, niraparib significantly extended progression free survival in two predefined populations, namely a patient population with altered homologous-recombination DNA repair pathways [i.e. homologous-recombination deficiency positive (HRd)] and the overall trial population. A prespecified exploratory subgroup analysis indicated that niraparib was also efficacious in patients who were homologous recombination deficiency negative or homologous recombination proficient (HRp). Niraparib has a manageable tolerability profile with myelosuppression as the main safety concern. Haematological reactions were managed with monitoring and dose reduction or interruption. A weight- and platelet count-based individualised dosage regimen introduced during the trial (and subsequently approved) appeared to improve haematological tolerability. Niraparib is a useful option for first-line maintenance therapy for advanced ovarian cancer in adults who responded to platinum-based chemotherapy, regardless of homologous-recombination deficiency status and is a promising option for HRp patients, for whom maintenance treatment options are limited.
\end{abstract}

\section{Plain Language Summary}

In 2021, ovarian cancer is predicted to be responsible for $\approx 43,770$ deaths in Europe and the USA combined. Niraparib $\left(Z_{e j u l a}{ }^{\mathrm{TM}}\right)$ is a once-daily oral treatment for first-line maintenance therapy in adults with advanced ovarian cancer that is responsive to chemotherapy. Although other agents of the same drug class (e.g. olaparib) are only approved for use against tumours with compromised DNA repair mechanisms, niraparib is approved without this restriction. Niraparib reduced the risk of disease progression or death in patients with newly diagnosed ovarian cancer, irrespective of whether the patients had compromised or functional DNA repair mechanisms. Abnormal blood counts are the main safety concern with niraparib, though adverse drug reactions may be managed through monitoring and interrupting or decreasing the dosage. Starting treatment at a personalised lower dosage may also reduce the likelihood of adverse drug reactions. Niraparib is a useful option for firstline maintenance therapy for advanced ovarian cancer, regardless of the patient's DNA repair mechanisms, and is a promising option for patients with functional DNA repair mechanisms, a group for which maintenance treatment options are limited.

Digital Features for this Adis Drug Evaluation can be found at https://doi.org/10.6084/m9.figshare.14913033.

The manuscript was reviewed by: J. Bieńkiewicz, Department of Operative Gynecology, Endoscopy and Gynecological Oncology, Polish Mother's Memorial Hospital - Research Institute, Lodz, Poland; S. Malander, Department of Clinical Sciences, Lund University, Lund, Sweden; K. Matsuo, Department of Obstetrics and Gynecology, University of Southern California, Los Angeles, CA, USA.

Arnold Lee

demail@springer.com

1 Springer Nature, Private Bag 65901, Mairangi Bay, Auckland 0754, New Zealand
Niraparib: clinical considerations in advanced ovarian cancer

PARP inhibitor with once-daily oral dosing for the maintenance treatment of advanced ovarian cancer

Extends progression free survival in adults with newly diagnosed advanced ovarian cancer, irrespective of HRD status

Manageable tolerability with a safety profile consistent with that established in other indications 


\section{Introduction}

Ovarian cancer is associated with the greatest number of deaths amongst gynaecological cancers in developed countries, with predicted estimates of 13,770 deaths in the USA and 30,000 deaths in the EU and UK in $2021[1,2]$. Although the overall 5-year survival rate for patients with invasive epithelial ovarian cancer is $48 \%$, many patients (64\%) are diagnosed with distant stage disease, for whom the 5-year survival rate is $31 \%$ [3]. Primary treatment for advanced epithelial ovarian cancer includes debulking surgery followed by platinum-based chemotherapy [4, 5]. Maintenance therapy with poly (ADP-ribose) polymerase (PARP) inhibitors is an established treatment option for recurrent ovarian cancers and, more recently, the use of PARP inhibitors has been extended to first-line maintenance therapy following successful primary treatment, with the aim of improving survival outcomes [6].

Homologous-recombination deficiency (HRD) is categorised as the presence of somatic or germline mutations of DNA repair genes (including mutations in $B R C A 1$ or 2, $P A L B 2, R A D 51 C$ and $A T M$ ), or genomic instability including loss of heterozygosity, telomeric allelic imbalance and large-scale state transitions [6]. Identifying patients who are HRD positive (HRd) or HRD negative [i.e. homologousrecombination proficient (HRp)] is relevant to treatment with PARP inhibitors, as inhibiting PARP-associated DNA repair is more effective in HRd patients due to the compromised state of DNA repair pathways. However, as $\approx 50 \%$ of patients with high-grade serous ovarian cancers do not show evidence of DNA damage [6], there has been an unmet need for therapies for HRp patients [7].

Niraparib (Zejula ${ }^{\mathrm{TM}}$ ) is a PARP inhibitor approved in the EU [8] and in the USA [9] for first-line maintenance therapy of advanced ovarian cancer, regardless of HRD status. It is also approved for use in certain patients as maintenance therapy for recurrent ovarian cancer $[8,9]$ and for treatment of advanced ovarian cancer after three or more chemotherapies [9]. This review will discuss the efficacy and tolerability of niraparib as first-line maintenance therapy for advanced ovarian cancer. The pharmacological properties of niraparib are summarised in Table 1. The discussion of niraparib for maintenance therapy in recurrent ovarian cancer (reviewed previously [10]) and other indications is outside the scope of this review.

\section{Therapeutic Efficacy of Niraparib}

The efficacy of niraparib as maintenance therapy for advanced ovarian cancer was investigated in the doubleblind, placebo-controlled, multicentre phase III PRIMA trial
(Fig. 1) [11]. Patients aged $\geq 18$ years with newly diagnosed, histologically confirmed advanced high-grade cancers of the ovary, peritoneum or fallopian tube who achieved an investigator-assessed complete or partial response to platinumbased chemotherapy were enrolled in this trial. Advanced tumours were those classified as stage III or IV according to International Federation of Gynecology and Obstetrics (FIGO) criteria; eligible stage III disease included inoperable tumours, or visible residual tumours following primary debulking surgery and no restrictions were placed for stage IV disease. Prior treatment with neoadjuvant chemotherapy was permitted regardless of stage [11].

Tumours were assessed for HRD status and HRd patients were analysed as a population in efficacy analyses (subsequently referred to as the HRd population) [11]. HRD was defined as the presence of a deleterious $B R C A$ gene mutation and/or a myChoice ${ }^{\circledR}$ test score of $\geq 42$ out of 100 (higher scores indicate higher levels of genomic abnormality). HRp patients or patients who had an undetermined HRD status were included in the overall population. Patient demographics at baseline were generally well balanced between the niarparib and placebo groups in the HRd population and in the overall population [11].

Patients were randomized to treatment with oral niraparib or placebo within 12 weeks of receiving their last dose of platinum-based chemotherapy $[11,12]$. Randomized treatment continued in 28-day cycles for 36 months; treatment could be discontinued due to patient or physician preference, unacceptable toxicity or disease progression. At the onset of the trial, niraparib was administered at a fixed dose of $300 \mathrm{mg}$ once daily. Following a protocol amendment to improve safety, the dosage of niraparib was reduced to $200 \mathrm{mg}$ once daily in patients with a body weight of $<77 \mathrm{~kg}$ and/or a platelet count of $<150,000$ platelets $/ \mu \mathrm{L}$ at baseline [11, 12].

The primary endpoint was progression-free survival (PFS), analysed hierarchically, first in the HRd population and in the overall population [11]. PFS was defined as the time from randomization to disease progression or death from any cause. Disease progression was determined by blinded central review using Response Evaluation Criteria in Solid Tumours (RECIST) version 1.1 criteria. Patients were assessed for disease progression every 12 weeks using magnetic resonance imaging or computed tomography, until treatment discontinuation [11].

Niraparib significantly $(p<0.001)$ extended PFS compared with placebo both in the HRd population and in the overall population (Table 2) [11]. The hazard ratios (HR) for disease progression or death favoured niraparib (HR $<1$ ) in both patient populations. PFS was also extended with niraparib versus placebo in several prespecified patient subgroups [exploratory analyses] (Table 3). Niraparib reduced the risk of disease progression or death relative to placebo 
Pharmacodynamic properties

Mechanism of action

Cardiovascular effects

Basic parameters

Proportional increase in $\mathrm{C}_{\max }$ and AUC with increasing niraparib dosage over a range of 30-400 mg; 2 to 3-fold accumulation following 21 days of niraparib 30-400 mg daily; $\mathrm{F} \approx 73 \%$; niraparib pharmacokinetics were not affected by a concomitant high-fat meal $[8,9]$

83.0\% plasma protein binding, mainly to albumin; apparent $\mathrm{V}_{\mathrm{d}} / \mathrm{F} 1074-1311 \mathrm{~L}[8,9]$; $\approx$ 3-fold higher niraparib exposure in tumours compared with plasma in a murine PDX tumour model [20]

Mean $\mathrm{t}_{1 / 2}$ of $36 \mathrm{~h}$ with multiple daily doses of niraparib $300 \mathrm{mg}$ [9]

Metabolised by carboxylesterases to an inactive metabolite, M1 [8, 9]; M1 is metabolised via glucuronidation [9]

$47.5 \%$ and $38.8 \%$ of a single $300 \mathrm{mg}$ dose of niraparib was excreted via renal and faecal routes (11\% and $19 \%$ unchanged drug) [9]

Special populations

No dosage adjustment is required for patients aged $\geq 65$ years or patients with mild hepatic impairment or mild to moderate chronic kidney disease; a reduced dosage of niraparib $200 \mathrm{mg}$ once daily is recommended in patients with moderate hepatic impairment. Data are limited in other populations $[8,9]$

Niraparib has the potential to cause embryonic or foetal harm $[8,9]$; contraception during niraparib treatment, and for 1 month (in the EU [8]) or 6 months (in the USA [9]) after treatment is recommended in women of childbearing age

Pharmacokinetic drug interactions

No formal clinical drug interaction studies are available with niraparib; as the drug weakly inhibits MATE 1 and 2 transporters, increased plasma concentrations of concomitantly administered MATE substrates cannot be excluded $[8,9]$

Potential pharmacokinetic drug interactions in the EU requiring caution
Niraparib may inhibit CYP3A4 in the gastrointestinal tract (but is not expected to inhibit CYP3A4 in the liver), and weakly induces CYP1A2, caution is advised with concomitant drugs metabolised by these enzymes with a narrow therapeutic index; niraparib very weakly inhibits P-gp and BCRP, and weakly inhibits OCT1, caution is recommended with concomitant drugs that are substrates for these transporters [8]

$A U C$ area under the plasma-time curve, $C_{\max }$ maximum plasma concentration, $C Y P$ cytochrome $\mathrm{P} 450, F$ absolute bioavailability, $H R$ homologous-recombinant, $I C_{50}$ half maximal inhibitory concentration, $P A R P$ poly(ADP-ribose) polymerase, $P D X$ patient-derived xenograft, $t_{1 / 2}$ half-life, $V_{d}$ volume of distribution

in $B R C A$ mutation $\mathrm{HRd}$, non-BRCA mutation HRd or HRp patients (Table 3) [11].

The efficacy of the fixed niraparib $300 \mathrm{mg}$ once daily dosage regimen was consistent with the individualised 200 or $300 \mathrm{mg}$ once daily dosage regimen, introduced later in the trial [13]. The HR for PFS in the niraparib versus placebo groups was 0.59 (95\% CI 0.46-0.76) in 475 patients receiving the fixed niraparib $300 \mathrm{mg}$ dose or placebo before the amendment and 0.69 (95\% CI 0.48-0.98) in 258 patients receiving an individualised niraparib dosage or placebo after the protocol amendment; PFS was not reported in these analyses. No significant treatment difference was reported between the fixed and individualised niraparib dosing subgroups [13].
Overall survival data were not mature at the time of the interim survival analysis, with only 79 deaths having occurred in in the overall population of 733 patients. The 24-month estimated Kaplan-Meier probabilities of survival with niraparib and placebo in the HRd population and in the overall population are reported in Table 2 [11].

\section{Tolerability of Niraparib}

Niraparib has a manageable tolerability profile as first-line maintenance therapy in patients with advanced ovarian cancer, consistent with that established in other indications [12]. Safety data are available for 484 niraparib and 244 placebo 


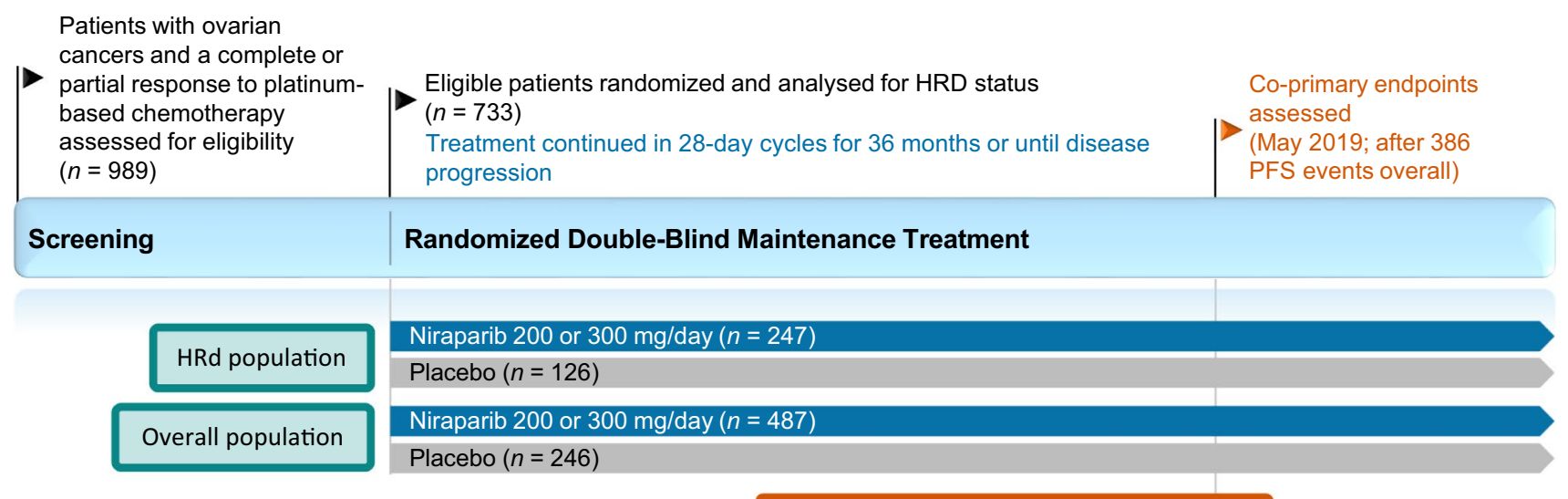

Median progression-free survival (months)

Fig. 1 Trial design of the pivotal phase III PRIMA trial, with efficacy reported in the animated figure (available online). Niraparib $300 \mathrm{mg}$ once daily was administered in 28-day cycles; this was later changed to 200 or $300 \mathrm{mg}$ once daily according to body weight or platelet count. HRd patients who were HRD positive, HRD homologousrecombination deficiency, $P F S$ progression-free survival recipients participating in the PRIMA trial (Sect. 2), where patients in the niraparib treatment arm were treated with a fixed niraparib dosage of $300 \mathrm{mg}$ once daily $(n=315)$ or an individualised niraparib dosage of 200 or $300 \mathrm{mg}$ once daily ( $n=169$ ) based on body weight and platelet count (described in Sect. 2) [11, 12]. Overall, adverse events (AEs) occurred more frequently with niraparib than placebo, which is consistent with other PARP inhibitors [11]. Grade $\geq 3$ treatment-related adverse events (TRAEs) were reported in $65.3 \%$ and $6.6 \%$ of patients, and serious TRAEs were reported in $24.4 \%$ and $2.5 \%$ of patients [11]. Treatment discontinuations due to AEs occurred in $12.0 \%$ and $2.5 \%$ of patients in the niraparaib and placebo groups [11]. Dosage reductions due to AEs were required in $70.9 \%$ of niraparaib recipients and $8.2 \%$ placebo recipients, and dosage interruptions due to AEs occurred in $79.5 \%$ and $18.0 \%$ of patients. [11]. No treatment-related deaths were reported during PRIMA $[11,12]$; two niraparib recipients $(0.4 \%)$ and one placebo recipient $(0.4 \%)$ died due to treatment-unrelated AEs [11].

Haematological events were the most commonly occurring grade $\geq 3$ AEs during PRIMA [11]. Grade $\geq 3$ AEs (defined as MedDRA terms) with an incidence $\geq 10 \%$ in niraparib recipients included anaemia (31.0\% of niraparib recipients and $1.6 \%$ of placebo recipients), thrombocytopenia $(28.7 \%$ and $0.4 \%)$, platelet count decreased $(13.0 \%$ and $0 \%$ ) and neutropenia (12.8\% and $1.2 \%)$. The majority of niraparib treatment discontinuations were due to myelosuppressive events, including thrombocytopenia $(4.3 \%)$, leukopenia $(2.1 \%)$, neutropenia $(1.9 \%)$ and anaemia $(1.9 \%)$; no placebo recipients discontinued treatment due to these events [11].

The effect of introducing an individualised dosing regimen (Sect. 2) on the incidence of grade $\geq 3$ treatment-emergent AEs (TEAEs) was analysed [12]. At the start of the trial, the incidence of any grade $\geq 3$ TEAEs in patients receiving a fixed niraparib dosage of $300 \mathrm{mg}$ once daily was $76 \%$ versus $60 \%$ in patients receiving individualised niraparib dosages of 200 or $300 \mathrm{mg}$ once daily. The incidence of grade $\geq 3$ events before and after the introduction of individualised niraparib dosages was $48 \%$ and $21 \%$ for thrombocytopenia, $36 \%$ and $23 \%$ for anaemia and $24 \%$ and $15 \%$ for neutropenia [12].

Adverse drug reactions (ADRs) which may require the discontinuation of niraparib therapy have been reported from PRIMA and other clinical trials of niraparib [8, 9]. Posterior reversible encephalopathy syndrome (PRES) was reported to occur during niraparib treatment at a frequency

Table 2 Efficacy of niraparib in the PRIMA phase III trial

\begin{tabular}{|c|c|c|c|c|}
\hline & \multicolumn{2}{|c|}{ HRd population } & \multicolumn{2}{|c|}{ Overall population } \\
\hline & $\begin{array}{l}\text { NIR }(n= \\
247)\end{array}$ & $\begin{array}{l}\text { PL }(n= \\
126)\end{array}$ & $\begin{array}{l}\text { NIR }(n= \\
487)\end{array}$ & $\begin{array}{l}\text { PL }(n= \\
246)\end{array}$ \\
\hline $\begin{array}{l}\text { Median PFS }{ }^{\mathrm{a}}[\mathrm{mo}] \\
(\mathrm{HR} ; 95 \% \mathrm{CI})\end{array}$ & $\begin{array}{l}21.9(0.43 ; \\
0.31-0.59)^{*}\end{array}$ & 10.4 & $\begin{array}{l}13.8(0.62 \\
0.50-0.76)^{*}\end{array}$ & 8.2 \\
\hline $\begin{array}{l}\text { 24-mo survival }{ }^{\mathrm{b}}[\%] \\
(\mathrm{HR} ; 95 \% \mathrm{CI})\end{array}$ & $\begin{array}{l}91(0.61 ; \\
0.27-1.39)\end{array}$ & 85 & $\begin{array}{l}84(0.70 ; 0.44 \\
1.11)\end{array}$ & 77 \\
\hline
\end{tabular}

Median follow-up duration at data cut-off (17 May 2019) was 13.8 mo and disease progression or death occurred in 154 patients in the HRd population and 386 patients in the overall population. Analyses were carried out in intention-to-treat populations [11].

$H R$ hazard ratio, $H R d$ patients who were homologous-recombination deficiency positive, mo months, NIR niraparib, PFS progression-free survival, $P L$ placebo

$* p<0.001$ vs PL

${ }^{\text {a}}$ Primary endpoint

${ }^{\mathrm{b}}$ Estimated Kaplan-Meier probability of overall survival 
Table 3 Prespecified exploratory analyses from the PRIMA trial [11]

\begin{tabular}{|c|c|c|c|}
\hline \multirow[b]{2}{*}{ Subgroup } & \multicolumn{2}{|l|}{ PFS (mo) } & \multirow[t]{2}{*}{$\mathrm{HR}^{\mathrm{a}}(95 \% \mathrm{CI})$} \\
\hline & $\operatorname{NIR}(n)$ & PL $(n)$ & \\
\hline HRp & $8.1(169)$ & $5.4(80)$ & $0.68(0.49-0.94)$ \\
\hline$B R C A$ mutation $\mathrm{HRd}$ & $22.1(152)$ & $10.9(71)$ & $0.40(0.27-0.62)$ \\
\hline Non- $B R C A$ mutation HRd & $19.6(95)$ & $8.2(55)$ & $0.50(0.31-0.83)$ \\
\hline Undetermined HRd status & NR (71) & NR (40) & $0.85(0.51-1.43)$ \\
\hline
\end{tabular}

$H R$ hazard ratio, $H R d$ patients who were homologous-recombination deficiency positive, HRp patients who were homologous-recombination deficiency negative, mo months, NIR niraparib, $N R$ not reported, $P F S$ progression-free survival, $P L$ placebo

${ }^{\mathrm{a}} \mathrm{HR}$ vs PL for disease progression or death

of $\geq 0.01 \%$ to $<0.1 \%$ in clinical trials [8]; however, no cases of PRES were reported during PRIMA [11]. Grade 3 or 4 hypertension ADRs were reported in $6 \%$ of niraparib recipients and $1 \%$ of placebo recipients during PRIMA, though $0 \%$ of niraparib recipients discontinued niraparib treatment due to hypertension ADRs. Monitoring blood pressure during niraparib treatment is recommended (Sect. 4) [8, 9].

Cases of myelodysplastic syndrome (MDS) or acute myeloid leukaemia (AML) have been reported during niraparib treatment, including one case of MDS in a niraparib recipient during PRIMA (no cases of AML were reported) [11]. Across all clinical trials investigating niraparib monotherapy, 15 cases of MDS or AML have been observed in 1785 niraparib recipients versus 3 cases in 488 patients receiving placebo or a treatment at the physician's discretion. Patients received 0.5 months to $>4.9$ years of niraparib treatment prior to developing MDS or AML [12].

\section{Dosage and Administration of Niraparib}

In the EU and USA, the recommended dosage of niraparib in adults as a monotherapy for maintenance treatment of advanced epithelial high-grade ovarian, fallopian tube or primary peritoneal cancer who achieved a complete or partial response to first-line platinum-based chemotherapy is niraparib $200 \mathrm{mg}$ once daily, taken orally [8, 9]. Niraparib $300 \mathrm{mg}$ once daily is recommended in patients with body weight $\geq 77 \mathrm{~kg}$, platelet count $\geq 150,000$ platelets $/ \mu \mathrm{L}$ and have mild or no hepatic impairment. Continuing niraparib treatment is recommended until unacceptable toxicity or disease progression [8, 9]. In the EU, niraparib is approved as a monotherapy for the maintenance treatment of adult patients with advanced epithelial (FIGO stages
III and IV) high-grade ovarian, fallopian tube or primary peritoneal cancer who are in complete or partial response following completion of first-line platinum-based chemotherapy [8]. In the USA, niraparib is approved as a firstline treatment for the same indication, though FIGO staging is omitted as a descriptor for advanced epithelial disease [9]. US prescribing information recommends initiating maintenance treatment for advanced ovarian cancer with niraparib no later than 12 weeks after the patient's most recent platinum-containing regimen. Niraparib should not be initiated in patients who have not recovered from haematological toxicity from prior chemotherapy [9].

Monitoring complete blood counts once weekly for the first month of treatment, monthly for the next 10 months (in the EU [8]) or 11 months (in the USA [9]) and periodically afterwards is recommended. Treatment interruption, dose reduction or treatment discontinuation may be necessary if haematological AEs are observed [8, 9]. In the EU, caution is recommended for concomitant administration of niraparib and anticoagulants or drugs which reduce thrombocyte count due to the risk of thrombocytopenia [8]. In the EU and the USA, blood pressure (and heart rate in the USA) should be monitored during niraparib treatment at least weekly for the first 2 months, monthly for the first year and periodically thereafter $[8,9]$. Close monitoring of patients with cardiovascular disorders is recommended in the USA [9].

Consult local prescribing information for warnings, use in special populations, drug interactions and recommended dosage adjustments for adverse reactions.

\section{Place of Niraparib in First-Line Maintenance Therapy for Advanced Ovarian Cancer}

The efficacy of niraparib as monotherapy for first-line maintenance treatment of advanced ovarian cancer was demonstrated in the phase III PRIMA trial (Sect. 2) [11]. PFS was significantly extended with niraparib versus placebo in both predefined patient populations, namely the HRd population and the overall population (Table 2). Furthermore, exploratory analyses indicated significant improvements in PFS with niraparib versus placebo in $B R C A$-related HRd, nonBRCA HRd and HRp patients (Table 3). Overall survival data were not mature at the time of the interim survival analysis (Sect. 2); and final overall survival results are awaited with interest. Niraparib had a manageable tolerability profile during the PRIMA trial, consistent with that seen in other indications (Sect. 3) [12]. Haematological events, which 
were the most commonly occurring AEs with niraparib, may be managed with monitoring and dosage reductions or interruptions [12]; $>70 \%$ of patients required dose reduction and $>70 \%$ of patients required treatment interruption due to an AE during PRIMA (Sect. 3). The incidence of grade $\geq 3$ haematological AEs was lower in patients who received platelet count- or weight-based individualised niraparib dosages of 200 or $300 \mathrm{mg}$ daily compared with a fixed niraparib dosage of $300 \mathrm{mg}$ daily [12]. The improved safety profile of the individualised starting dosage is reflected in the approved dosing regimen of niraparib (Sect. 4). The introduction of the individualised niraparib dosage during the PRIMA trial was not associated with a significant difference in efficacy (Sect. 2) [13]. However, as the PRIMA trial was not powered or designed for the individualised niraparib dosage regimen, a reduction in efficacy with niraparib $200 \mathrm{mg}$ daily cannot be excluded [12].

Current guidelines recommend niraparib as a treatment option in patients with advanced epithelial high-grade ovarian cancer who responded to platinum-based chemotherapy $[5,7,14]$. The UK National Institute for Health and Care Excellence (NICE) recommends the use of niraparib under the Cancer Drugs Fund for patients with FIGO stage III or IV cancers who responded to platinum-based chemotherapy [7]. US National Comprehensive Cancer Network (NCCN) recommendations for patients with stage II-IV ovarian cancer who achieved a partial or complete response to platinum-based chemotherapy vary based on whether the patient received bevacizumab as part of their primary therapy [5]. In patients not previously treated with bevacizumab, niraparib is recommended as a treatment option regardless of $B R C A 1$ or 2 mutation status. In patients who have been previously treated with bevacizumab, niraparib is a recommended option for those with $B R C A 1$ or 2 mutations but not for patients with wild-type or unknown BRCAl or 2 mutation status. For all patients, the recommended treatment duration with niraparib is 36 months or until disease progression or unacceptable toxicity [5]. The American Society of Clinical Oncology (ASCO) recommends niraparib as a treatment option in all women who achieved a partial or complete response to platinum-based chemotherapy for stage III or IV high-grade serous or endometrioid ovarian cancer [14]. The recommended treatment duration is 3 years, which may be extended if the patient is continuing to derive clinical benefit [14]. Although the maximum treatment duration and the recommended FIGO stages of patients is specified in the NCCN and ASCO guidelines, the US prescribing information does not state a maximum treatment duration and the approved indication is not restricted by FIGO stage (Sect. 4) [9]. European Society for Medical Oncology and European Society of Gynaecologial Oncology guidelines for ovarian cancer are yet to be updated to include the approval of niraparib in this indication [4].
No direct comparisons of efficacy or safety between niraparib and other approved agents for the first-line maintenance therapy of advanced epithelial high-grade ovarian cancer, including olaparib, have been performed $[15,16]$. Unlike olaparib, which is approved for use in patients with deleterious or suspected $B R C A$ mutations only $[15,16]$, niraparib is not restricted by HRD status. This distinction of HRD status between olaparib and niraparib is reflected in NCCN and ASCO guidelines for ovarian cancer, with olaparib monotherapy recommended in patients with $B R C A$ mutations only [5, 14]. A potential advantage of niraparib is once-daily dosing (Sect. 4), which may be more convenient for patients than the twice-daily dosage regime of olaparib [15].

Cost-effectiveness data for niraparib as maintenance therapy are limited, especially as overall survival data are not mature. One US-based analysis compared the costeffectiveness of niraparib maintenance therapy against observation, using data from the PRIMA trial [17]. The baseline model assumed the overall survival was 3-times longer than PFS across all patients with a health state utility of 0.75 . The costs of niraparib toxicity were calculated as the treatment cost for anaemia as it was the most common grade 3-4 adverse event. Incremental cost-effectiveness ratios (ICERs) were below the willingness-to-pay threshold of US $\$ 100,000$ per quality-adjusted life-year (QALY) for all patient populations; the HRd population (US \$56,329 ICER per QALY), overall trial population (US \$72,829), $B R C A$-HRd (US \$58,348), non BRCA-HRd (US \$50,914) or HRp (US $\$ 88,741$ ) patients. In sensitivity analyses of the overall trial population, niraparib maintenance therapy remained cost-effective while the overall survival was $\geq 2$.2-times longer than PFS or if the health state utility was $>0.54$ [17]. In the UK, niraparib is recommended for use via the Cancer Drugs Fund; niraparib is not recommended for widespread use by the UK NHS as ICERs were outside the range required to demonstrate cost-effectiveness (ICERs were not reported) [7]. As with all pharmacoeconomic analyses, these analyses are subject to limitations, including the use of uncertain estimates of overall survival with niraparib maintenance therapy. Mature survival data may allow for more robust analyses in the future [7, 17].

In summary, significant extensions in PFS were observed in the PRIMA trial with niraparib compared with placebo in HRd patients, and in the overall population. Haematological reactions, the main safety concern with niraparib, were managed with monitoring and dosage modifications or dose interruption. Thus, niraparib is a useful option for the first-line maintenance therapy of advanced ovarian cancer in adults who responded to platinum-based chemotherapy and may be a particularly promising option for HRp patients, for whom maintenance treatment options are limited. 


\section{Data Selection Niraparib: 271 records identified}

Duplicates removed

Excluded during initial screening (e.g. press releases; news reports; not relevant drug/indication; preclinical study; reviews; case reports; not randomized trial)

Excluded during writing (e.g. reviews; duplicate data; small patient number; nonrandomized/phase I/II trials)

Cited efficacy/tolerability articles Cited articles not efficacy/tolerability

Search Strategy: EMBASE, MEDLINE and PubMed from 2018 to present. Previous Adis Drug Evaluation published in 2018 was hand-searched for relevant data. Clinical trial registries/ databases and websites were also searched for relevant data. Key words were niraparib, Zejula, epithelial ovarian cancer, peritoneal cancer, fallopian tube cancer, maintenance therapy. Records were limited to those in English language. Searches last updated 1 Sept 2021

Supplementary Information The online version contains supplementary material available at https://doi.org/10.1007/s11523-021-00841-2.

Acknowledgements During the peer review process, the manufacturer of niraparib was also offered an opportunity to review this article. Changes resulting from comments received were made on the basis of scientific and editorial merit.

\section{Declarations}

Funding The preparation of this review was not supported by any external funding.

Authorship and conflict of interest Arnold Lee is a salaried employee of Adis International Ltd/Springer Nature, and declares no relevant conflicts of interest. All authors contributed to the review and are responsible for the article content.

Ethics approval, Consent to participate, Consent to publish, Availability of data and material, Code availability Not applicable.

Open Access This article is licensed under a Creative Commons Attribution-NonCommercial 4.0 International License, which permits any non-commercial use, sharing, adaptation, distribution and reproduction in any medium or format, as long as you give appropriate credit to the original author(s) and the source, provide a link to the Creative Commons licence, and indicate if changes were made. The images or other third party material in this article are included in the article's Creative Commons licence, unless indicated otherwise in a credit line to the material. If material is not included in the article's Creative Commons licence and your intended use is not permitted by statutory regulation or exceeds the permitted use, you will need to obtain permission directly from the copyright holder. To view a copy of this licence, visit http://creativecommons.org/licenses/by-nc/4.0/.

\section{References}

1. Carioli G, Malvezzi M, Bertuccio P, et al. European cancer mortality predictions for the year 2021 with focus on pancreatic and female lung cancer. Ann Oncol. 2021;32(4):478-87.

2. American Cancer Society. Cancer facts \& figures 2021. 2021. https://www.cancer.org/. Accessed 1 Sept 2021.

3. American Cancer Society. Cancer facts \& figures 2018 special section: ovarian cancer. 2018. https://www.cancer.org/. Accessed 1 Sept 2021.

4. Colombo N, Sessa C, Bois AD, et al. ESMO-ESGO consensus conference recommendations on ovarian cancer: pathology and molecular biology, early and advanced stages, borderline tumours and recurrent disease. Int J Gynecol Cancer. 2019;29(4):728-60.

5. National Comprehensive Cancer Network. Ovarian cancer including fallopian tube cancer and primary peritoneal cancer (NCCN guidelines). 2021. http://www.nccn.org/. Accessed 1 Sept 2021.

6. Kurnit KC, Fleming GF, Lengyel E. Updates and new options in advanced epithelial ovarian cancer treatment. Obstet Gynecol. 2021;137(1):108-21.

7. National Institute for Health and Care Excellence. Niraparib for maintenance treatment of advanced ovarian, fallopian tube and peritoneal cancer after response to first-line platinum-based chemotherapy. 2021. http://www.nice.org.uk. Accessed 1 Sept 2021.

8. European Medicines Agency. Zejula (niraparib): summary of product characteristics. 2021. https://www.ema.europa.eu/. Accessed 1 Sept 2021.

9. GlaxoSmithKline. Zejula (niraparib): US prescribing information. 2021. https://www.zejula.com/. Accessed 1 Sept 2021.

10. Heo Y-A, Duggan ST. Niraparib: a review in ovarian cancer. Target Oncol. 2018;13(4):533-9.

11. González-Martín A, Pothuri B, Vergote I, et al. Niraparib in patients with newly diagnosed advanced ovarian cancer. N Engl J Med. 2019;381(25):2391-402.

12. European Medicines Agency. Assessment report: Zejula (niraparib). 2020. https://www.ema.europa.eu/. Accessed 1 Sept 2020.

13. Mirza MR, Gonzalez Martin A, Graybill W, et al. Evaluation of an individualized starting-dose of niraparib in the PRIMA/ENGOTOV26/GOG-3012 study [abstract no. 6050]. In: ASCO 2020.

14. Tew WP, Lacchetti C, Ellis A, et al. PARP inhibitors in the management of ovarian cancer: ASCO guideline. J Clin Oncol. 2020;38(30):3468-93.

15. AstraZeneca Pharmaceuticals LP. Lynparza (olaparib): US prescribing information. 2021. https://www.lynparza.com/. Accessed 1 Sept 2021.

16. European Medicines Agency. Lynparza (olaparib): summary of product characteristics. 2021. https://www.ema.europa.eu/. Accessed 1 Sept 2021.

17. Barrington DA, Tubbs C, Smith HJ, et al. Niraparib maintenance in frontline management of ovarian cancer: a cost effectiveness analysis. Int J Gynecol Cancer. 2020;30(10):1569-75.

18. Jones P, Altamura S, Boueres J, et al. Discovery of 2-\{4-[(3S)piperidin-3-yl]phenyl\}-2H-indazole-7-carboxamide (MK-4827): a novel oral poly(ADP-ribose)polymerase (PARP) inhibitor efficacious in BRCA-1 and -2 mutant tumors. J Med Chem. 2009;52(22):7170-85.

19. AlHilli MM, Becker MA, Weroha SJ, et al. In vivo anti-tumor activity of the PARP inhibitor niraparib in homologous recombination deficient and proficient ovarian carcinoma. Gynecol Oncol. 2016;143(2):379-88.

20. Sun K, Mikule K, Wang Z, et al. A comparative pharmacokinetic study of PARP inhibitors demonstrates favorable properties for niraparib efficacy in preclinical tumor models. Oncotarget. 2018;9(98):37080-96. 